\title{
El "residente como docente": estudio fenomenográfico sobre las experiencias docentes en residentes de Medicina Familiar de una Universidad en Chía, Colombia
}

\section{The "resident as teacher": a phenomenographic study about the teaching experiences in Family Medicine residents at a University in Chía, Colombia}

\author{
Adriana Marcela Rincón-Salazar ${ }^{1}$, Francisco Lamus-Lemus ${ }^{2 *}$, Natalia Reinoso Chávez ${ }^{3}$ y \\ Claudia Liliana Jaimes-Peñuela ${ }^{4}$ \\ 1 Maestría en Educación Médica, Facultad de Medicina, Universidad de La Sabana, Chía, Colombia; \\ adriana.rincon1@unisabana.edu.co, 0000-0002-0124-5144 \\ 2 Facultad de Medicina, Universidad de La Sabana, Chía, Colombia. francisco.lamus@unisabana.edu.co, \\ 0000-0002-1086-2945 \\ 3 Facultad de Medicina, Universidad de La Sabana, Chía, Colombia; natalia.reinoso@unisabana.edu.co, 0000- \\ 0002-2189-6155 \\ 4 Facultad de Medicina, Universidad de La Sabana, Chía, Colombia; claudia.jaimes2@unisabana.edu.co , \\ 0000-0002-5240-4942 \\ * Correspondencia: francisco.lamus@unisabana.edu.co
}

Recibido: 05/08/2021; Aceptado: 26/10/21; Publicado: 2/11/21

Resumen: Los residentes médicos tienen un rol fundamental en el proceso de formación de estudiantes, internos y co-residentes, con labores implícitas de docencia en sus obligaciones como estudiantes de posgrado y sin ningún tipo de formación para ello. Previo a la implementación de programas de "residente como docente", resulta útil indagar sobre las experiencias docentes de los residentes para el desarrollo de un currículo acorde a las características y necesidades del medio. Este estudio cualitativo con enfoque fenomenográfico realizado en 15 residentes de Medicina Familiar de una Universidad colombiana buscó identificar las variaciones en las experiencias docentes de los residentes. Tras el análisis de los datos, se identificaron 5 categorías de descripción relacionadas de manera jerárquica y determinadas a partir de 7 dimensiones de variación. Las formas identificadas de experimentar el rol del "residente como docente" fueron el "transmisor pasivo impuesto", el "facilitador del conocimiento", el "facilitador de la práctica clínica", el "role model" y el "lifelong learner". Estas categorías se encuentran interconectadas y cuentan el potencial de avanzar hacia las categorías de mayor comprensión del rol con los estímulos y recursos adecuados. Lo anterior puede ser utilizado como sustrato para el desarrollo de un programa de "residente como docente"; situación deseable en el contexto latinoamericano donde los residentes tienen diversas responsabilidades docentes.

Palabras clave: Residencia Médica; Educación médica; Medicina.

Abstract: Medical residents have a fundamental role in the training process of students, interns and peer-residents, with implicit teaching tasks in their obligations as postgraduate students and without any type of training for it. Prior to the implementation of a "resident as teacher" program, it is useful to inquire about the teaching experiences of residents for the development of a curriculum according to the characteristics and needs of the environment. This qualitative study with a phenomenographic approach carried out in 15 Family Medicine residents from a Colombian University sought to identify the variations in the teaching experiences of the residents. After the data analysis, 5 categories of description related in a hierarchical way and determined from 7 dimensions of variation were identified. The identified ways of experiencing the role of the "resident as teacher" were the "imposed passive transmitter", the "knowledge facilitator", the "clinical practice facilitator", the "role model" and the "lifelong learner". These 
categories are interconnected and have the potential to advance towards the categories of greater understanding of the role with the appropriate stimuli and resources. The foregoing can be used as a substrate for the development of a "resident as teacher" program; a desirable situation in the Latin American context where residents have diverse teaching responsibilities.

Keywords: Internship and Residency; Medical education; Medicine.

\section{Introducción}

Los residentes médicos representan un rol fundamental en el proceso de formación de estudiantes, internos y co-residentes, con labores implícitas de docencia en sus obligaciones como estudiantes de posgrado y sin ningún tipo de formación para ello (1-2). La importancia de este rol radica en la gran cantidad de tiempo que los residentes pasan con los estudiantes y la supervisión directa que les proporcionan (3-4). Pese a ello y aunque son los encargados de enseñar gran parte del currículo informal, la evidencia muestra que lo hacen de manera inefectiva (3).

Las actividades y escenarios docentes del residente abarcan una miríada de posibilidades (5-6). Aunque estas han sido ampliamente caracterizadas en países norteamericanos y europeos con miras a la creación de programas de "residente como docente", en Colombia son pocos los estudios que existen al respecto, lo cual dificulta el diseño de programas de formación docente dirigidos a residentes $(2,5,7)$.

Es un hecho que previo a la implementación de programas de "residente como docente", resulta útil indagar sobre las experiencias docentes en contexto, para así desarrollar currículos acordes con las características y necesidades del medio (7). En la publicación realizada por Mann, Suton y Frank sobre los aspectos que se deben tener en cuenta al implementar un programa de formación docente para residentes, se resalta la necesidad de conocer las actividades de docencia que realizan los residentes (7).

Por lo anterior, este estudio buscó indagar sobre las variaciones en las experiencias docentes de residentes de Medicina Familiar de una Universidad colombiana, para así generar repercusiones en el diseño de un programa de "residente como docente" dirigido a los especialistas en formación de esta y otras instituciones conexas. Cabe resaltar que, si bien en algunos estudios realizados en docentes médicos se ha explorado la percepción del rol docente de acuerdo con diversos determinantes como el actual ambiente educacional y el nivel de entrenamiento como profesor, no se ha investigado la variación en las experiencias del "residente como docente" ni tampoco han sido enmarcados por categorías en la literatura (8-9). Ahora bien, con el fin de tener una mejor comprensión de las características de cada una de las categorías de descripción propuestas, se decidió contrastarlas con los roles de profesores descritos en la literatura médica, con un particular énfasis en los 12 roles docentes señalados por The Association for Medical Education in Europe (AMEE) en su guía número 20 (9).

\section{Métodos}

Este estudio corresponde a una investigación cualitativa con un enfoque fenomenográfico. Los estudios fenomenográficos tienen una larga tradición en las investigaciones en educación y en salud. En el ámbito de la educación médica, se ha incrementado su uso puesto que se basan en la asunción de que casi cualquier fenómeno se puede comprender en un número limitado de formas, además de ser utilizados como herramienta de aprendizaje y para el desarrollo de competencias (10-14). 
El resultado de un estudio fenomenográfico está dispuesto en un conjunto ordenado de categorías que con frecuencia representan diferentes niveles de entendimiento del fenómeno (15-16). Seleccionamos este abordaje dado que estábamos interesados en hacer un mapeo cualitativo del rango completo de experiencias posibles de los residentes en torno a su rol docente y como se relacionan entre sí.

La población de estudio fueron residentes de Medicina Familiar en cualquiera de los 3 años de formación (tabla 1). El entrenamiento de los residentes se facilita en escenarios de consulta externa, hospitalización, urgencias, visita domiciliaria y comunidad, en el área rural y urbana, en hospitales de contratación pública y privada; durante el tiempo de preparación no hay una educación formal en docencia. La selección de los participantes se hizo mediante muestreo en bola de nieve.

\begin{tabular}{|c|c|c|c|}
\hline \multicolumn{2}{|c|}{ Año de residencia } & \multicolumn{2}{|l|}{ Estado civil } \\
\hline R1 (primer año) & 3 & Soltero/soltera & 7 \\
\hline R2 (segundo año) & 6 & Unión libre & 3 \\
\hline R3 (tercer año) & 6 & Casado/casada & 5 \\
\hline \multicolumn{2}{|c|}{ Universidad de procedencia } & Separado/separada & 0 \\
\hline $\begin{array}{c}\text { Universidad de este } \\
\text { estudio }\end{array}$ & 10 & \multicolumn{2}{|l|}{ Hijos } \\
\hline Otras universidades & 5 & $\mathrm{Si}$ & 3 \\
\hline \multicolumn{2}{|c|}{$\begin{array}{l}\text { Años de trabajo previo al inicio de la } \\
\text { residencia médica }\end{array}$} & No & 12 \\
\hline Menos de 1 año & 0 & \multicolumn{2}{|c|}{$\begin{array}{c}\text { Trabajo simultáneo durante } 1 \mathrm{c} \\
\text { residencia }\end{array}$} \\
\hline 1-3 años & 13 & $\mathrm{Si}$ & 4 \\
\hline 3-5 años & 0 & No & 11 \\
\hline$>5$ años & 2 & \multicolumn{2}{|c|}{$\begin{array}{c}\text { Deseo manifiesto de carrera } \\
\text { docente en el futuro }\end{array}$} \\
\hline $\begin{array}{c}\text { Estudios previos en } \\
\text { docencia }\end{array}$ & 0 & $\mathrm{Si}$ & 6 \\
\hline $\begin{array}{c}\text { Estudios adicionales de } \\
\text { posgrado }\end{array}$ & 0 & No & 9 \\
\hline
\end{tabular}

Previo a la firma del consentimiento informado por los participantes y una vez garantizados los 7 requisitos éticos de investigación en humanos planteados por Ezequiel Emanuel (17), además de contar con el aval por la comisión de investigación de la Universidad, se realizaron 15 entrevistas que fueron audio-grabadas para su posterior transcripción y análisis. Si bien en el enfoque fenomenográfico se ha descrito requerir entre 15 y 30 participantes, la mayoría de los estudios logran identificar la totalidad de variación de un fenómeno con 15 participantes (12-13).

Para la recolección de la información, se diseñó una guía de entrevista semiestructurada individual que facilitara al participante expresar su relación con el fenómeno. Cada entrevista tuvo continuidad hasta que el entrevistador y el participante consideraron que se habían discutido todos los aspectos del fenómeno. El análisis de los datos se realizó a partir de los 7 pasos para el análisis fenomenográfico descrito por 
Dahlgren y Fallsberg (tabla 2); inicialmente se realizó de forma manual, con el posterior uso del software NVivo versión 12 como soporte informático.

Se utilizaron múltiples medidas para garantizar el rigor de la investigación, incluida la identificación de las preconcepciones del fenómeno por parte de los investigadores, el uso de facilitadores para la expresión genuina del fenómeno, co-investigadores y consenso de negociación, y los criterios primarios para juzgar la calidad de las categorías de descripción descritos por Marton y Booth (tabla 3) (18).

\begin{tabular}{|l|l|}
\hline \multicolumn{2}{|c|}{ Tabla 2. Siete pasos para el análisis de información } \\
\hline 1. Familiarización & $\begin{array}{l}\text { Leer todas las transcripciones, familiarizarse con los datos empíricos y } \\
\text { obtener un sentido del todo. }\end{array}$ \\
\hline 2. Condensación & $\begin{array}{l}\text { Identificar las unidades de significado con el propósito de un examen } \\
\text { profundo posterior. Se busca un aspecto central en las respuestas. }\end{array}$ \\
\hline 3. Comparación & $\begin{array}{l}\text { Comparar las unidades en cuanto a similitudes y diferencias. El } \\
\text { investigador procura delimitar las categorías. }\end{array}$ \\
\hline 4. Agrupación & $\begin{array}{l}\text { Colocar en un mismo grupo las respuestas que expresen maneras similares } \\
\text { de entender el fenómeno. }\end{array}$ \\
\hline 5. Articulación & Capturar el significado esencial de cada categoría \\
\hline 6. Etiquetar & $\begin{array}{l}\text { Expresar el significado central de la categoría } \\
\text { Los pasos 3-6 se repiten de manera iterativa para asegurar que las } \\
\text { similitudes y las diferencias entre categorías se distingan y formulen de } \\
\text { manera distinta }\end{array}$ \\
\hline 7. Contrastar & Compara las categorías en cuanto a similitudes y diferencias \\
\hline Basado en las referencias 16,27,29. \\
\hline
\end{tabular}

Tabla 3. Criterios primarios para juzgar la calidad de las categorías de descripción.

1 Cada categoría de descripción revela un aspecto diferente de la manera de experimentar el fenómeno.

2 Las categorías tienen una relación lógica y clara entre sí; en este estudio están relacionas de manera jerárquica.

3 Los resultados finales son parsimoniosos, es decir que son presentados en la menor cantidad de categorías posibles.

Basado en la referencia 18.

\section{Resultados}

Se identificaron 5 categorías de descripción o maneras de experimentar el rol del "residente como docente" relacionadas de manera jerárquica y delimitadas a partir de 7 dimensiones de variación; estas dimensiones hicieron referencia a los aspectos críticos que determinaron las diferentes formas de experimentar el fenómeno y permitieron vincular las categorías entre sí (Tabla 4). Tras un consenso de negociación entre los investigadores, se estableció que las diferentes maneras jerárquicas de experimentar el rol del "residente como docente" fueron el transmisor pasivo impuesto, el facilitador del conocimiento, el facilitador de la práctica clínica, el role model y el "lifelong learner" (Figura 1).

\section{El "residente como docente" transmisor pasivo impuesto}

Ubicado en el primer escalón de la jerarquía de experiencia del rol, el "transmisor pasivo impuesto" ejerce actividades docentes centradas en el profesor mediante relaciones pedagógicas verticales, en escenarios percibidos como muy favorables para este fin, es decir actividades planeadas en las cuales se le solicita de forma explícita ejercer un rol de profesor. No obstante, no se percibe a sí mismo como un docente, identificando barreras 
intrínsecas para ejercer el rol, principalmente el temor a ser avergonzados por no contar con el conocimiento médico suficiente.

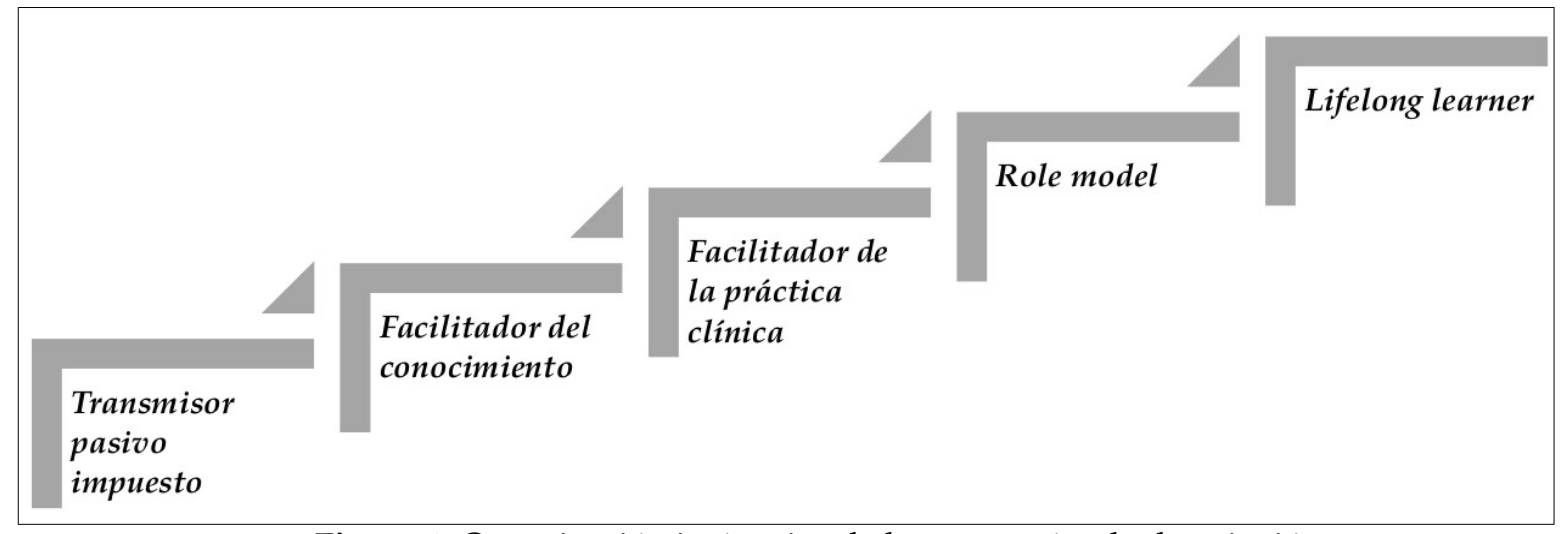

Figura 1. Organización jerárquica de las categorías de descripción.

El "residente como docente" facilitador del conocimiento

Posicionado en el segundo escalón de la jerarquía, el "facilitador del conocimiento" utiliza estrategias docentes centradas en el estudiante, con una relación pedagógica horizontal. Desempeña su rol docente en aquellos escenarios percibidos como muy favorables o favorables y su principal motivación para desempeñarse como docente es el gusto por la labor. Se percibe a sí mismo como un docente en potencia que evolucionará en el rol durante la residencia a medida que avanza en su proceso de formación. La característica esencial de esta categoría es su enfoque en los aspectos teóricos del ejercicio médico, con un amplio interés en la transmisión de un -saber cómo-.

\section{El "residente como docente" facilitador de la práctica clínica}

Ubicado en el tercer escalón de la jerarquía, el "facilitador de la práctica clínica", al igual que en la categoría anterior, utiliza estrategias docentes centradas en el estudiante, con una relación pedagógica horizontal. Desempeña su rol docente en aquellos escenarios percibidos incluso como poco favorables, descritos en este estudio como los escenarios clínicos bajo presión, situación ampliamente relacionada con la finalidad pedagógica de esta categoría centrada en el -hacer- del ejercicio médico. En adición, su principal motivación para ejercer la docencia es el entendimiento de esta como una tarea fundamental en el propio proceso de aprendizaje. Se percibe a sí mismo como un docente en potencia que evolucionará en el rol durante la residencia.

\section{El "residente como docente" role model}

Posicionado en el cuarto escalón de la de la jerarquía de experiencias se encuentra el "role model". Esta categoría utiliza estrategias pedagógicas centradas en el estudiante, con una relación pedagógica horizontal con el aprendiz y el desarrollo del ejercicio docente incluso en escenarios percibidos como desfavorables para esta labor. La principal motivación es un sentimiento de responsabilidad con el estudiante, en contexto de una comprensión del rol docente como inherente a la labor del residente. 


\begin{tabular}{|c|c|c|c|c|c|c|c|}
\hline \multirow[b]{2}{*}{$\begin{array}{l}\text { Categorías de } \\
\text { descripción }\end{array}$} & \multicolumn{7}{|c|}{ Dimensiones de variación } \\
\hline & $\begin{array}{c}\text { Finalidad } \\
\text { pedagógica } \\
\text { (pirámide de } \\
\text { Miller) } \\
\end{array}$ & $\begin{array}{c}\text { Estrategia } \\
\text { pedagógica }\end{array}$ & $\begin{array}{c}\text { Relación } \\
\text { pedagógica }\end{array}$ & $\begin{array}{l}\text { Escenarios } \\
\text { docentes }\end{array}$ & Motivación & Barrera & $\begin{array}{c}\text { Propia percepción } \\
\text { docente }\end{array}$ \\
\hline $\begin{array}{c}\text { Transmisor pasivo } \\
\text { impuesto }\end{array}$ & Saber & $\begin{array}{c}\text { Centrada en el } \\
\text { profesor }\end{array}$ & Vertical & $\begin{array}{c}\text { Muy } \\
\text { favorables }\end{array}$ & Extrínseca & $\begin{array}{c}\text { Intrínseca: } \\
\text { miedo }\end{array}$ & $\begin{array}{c}\text { No se ve como } \\
\text { docente }\end{array}$ \\
\hline $\begin{array}{l}\text { Facilitador del } \\
\text { conocimiento }\end{array}$ & Saber cómo & $\begin{array}{l}\text { Centrada en el } \\
\text { estudiante }\end{array}$ & Horizontal & Favorables & Intrínseca: gusto & Extrínseca & $\begin{array}{l}\text { Rol evolutivo como } \\
\text { docente durante la } \\
\text { residencia }\end{array}$ \\
\hline $\begin{array}{l}\text { Facilitador } \\
\text { de la práctica } \\
\text { clínica }\end{array}$ & Hacer & $\begin{array}{c}\text { Centrado en el } \\
\text { estudiante }\end{array}$ & Horizontal & $\begin{array}{c}\text { Poco } \\
\text { favorables }\end{array}$ & $\begin{array}{l}\text { Intrínseca: parte del } \\
\text { proceso académico } \\
\text { propio }\end{array}$ & Extrínseca & $\begin{array}{c}\text { Rol evolutivo como } \\
\text { docente durante la } \\
\text { residencia }\end{array}$ \\
\hline Role model & Ser & $\begin{array}{c}\text { Centrado en el } \\
\text { estudiante }\end{array}$ & Horizontal & $\begin{array}{c}\text { No } \\
\text { favorables }\end{array}$ & $\begin{array}{c}\text { Intrínseca: } \\
\text { responsabilidad con } \\
\text { el estudiante }\end{array}$ & Extrínseca & $\begin{array}{l}\text { Rol docente es } \\
\text { inherente a la } \\
\text { residencia }\end{array}$ \\
\hline Lifelong learner & Ser & $\begin{array}{l}\text { Centrado en el } \\
\text { estudiante }\end{array}$ & Horizontal & $\begin{array}{c}\text { No } \\
\text { favorables }\end{array}$ & $\begin{array}{c}\text { Intrínseca: } \\
\text { responsabilidad social } \\
\text { e historial de anti-role } \\
\text { model en su } \\
\text { pregrado }\end{array}$ & Extrínseca & $\begin{array}{l}\text { Responsabilidad } \\
\text { docente con } \\
\text { metacognición del } \\
\text { ejercicio }\end{array}$ \\
\hline
\end{tabular}




\section{El "residente como docente" lifelong learner}

Ubicado en el último escalón de la jerarquía de experiencias se encuentra el "lifelong learner"; esta categoría es similar a la anterior, con una estrategia pedagógica centrada en el estudiante, relaciones pedagógicas de naturaleza horizontal y un desarrollo del ejercicio docente incluso en escenarios percibidos como desfavorables. Sin embargo, existen dos características diferenciadoras propias de esta categoría: la motivación intrínseca determinada por la responsabilidad social y un historial de anti-role model durante el propio proceso de formación, en adición a la metacognición del ejercicio docente.

Cabe señalar que las categorías no se vieron influenciadas por el nivel de formación actual de los residentes. Además, ninguno de los participantes había recibido entrenamiento formal en docencia ni contaba con otro tipo de educación de posgrado. En la Tabla 5 se resaltan algunos comentarios significativos de cada categoría de descripción.

\section{Discusión}

Al finalizar esta investigación se estableció que las variaciones en las experiencias del "residente como docente", están determinadas por la interacción de factores personales y contextuales, además de la propia concepción del rol docente. Se hace necesario resaltar que las categorías de descripción se encuentran interconectadas, con el potencial de avanzar hacia la siguiente categoría (con los incentivos y recursos adecuados). A continuación, se discuten las categorías en detalle.

\section{El "residente como docente" transmisor pasivo impuesto}

$\mathrm{Al}$ contrastar los resultados de esta categoría con lo reportado en la literatura sobre perfiles docentes, en el estudio realizado por Jacobs en el 2014, se identificaron 5 perfiles de maestros en el campo de la educación médica: "transmisores", "organizadores", "intermedios", "facilitadores" y "agentes de cambio conceptual". En los "transmisores" al igual que en esta categoría, sobresalió la preferencia por las estrategias pedagógica centradas en el docente, explicado por una apreciación muy baja del aprendizaje activo y una escasa orientación a la práctica profesional, sumado a la carencia de habilidades pedagógicas (19).

Por otra parte, en el estudio realizado por Trigwell en 1999 sobre las percepciones de docentes universitarios con respecto a su enfoque de enseñanza, los maestros que describieron su enfoque docente como "transmisión de la información/centrada en el profesor" se relacionó con una mayor probabilidad de que sus estudiantes utilizaran estrategias de aprendizaje orientadas a un aprendizaje superficial (20). Se debe comprender que esto representa una problemática para la educación, puesto que en la literatura se ha reportado de forma consistente que los abordajes de aprendizaje superficial se relacionan con resultados de aprendizaje de muy baja calidad (20).

\section{El "residente como docente" facilitador del conocimiento}

De acuerdo con la guía 20 de AMEE los roles docentes están agrupados en 12 categorías determinados por 6 áreas de actividad (9). Al comparar dichos roles con lo evidenciado en este estudio, se encuentran similitudes entre el "facilitador del conocimiento" y el "facilitador". Este se entiende como un administrador del aprendizaje de los estudiantes, en donde su rol no es informar, sino alentarlos y apoyarlos en su proceso de aprendizaje mediante la utilización de problemas teóricos; esto resalta el abordaje constructivista del aprendizaje utilizado en esta categoría, en cuanto reconoce y avanza sobre la trayectoria y el conocimiento previo que traen los participantes (9). 


\begin{tabular}{|c|c|}
\hline \multicolumn{2}{|r|}{ Tabla 5. Comentarios significativos de las categorías de descripción } \\
\hline $\begin{array}{l}\text { Categoría de } \\
\text { descripción }\end{array}$ & Comentario significativo \\
\hline $\begin{array}{c}\text { Transmisor } \\
\text { pasivo } \\
\text { impuesto }\end{array}$ & $\begin{array}{l}\text { “...siempre estaba uno con la responsabilidad que tenía uno el profe ahí al lado y el profe } \\
\text { le decía, usted pilas que usted tiene que estar pendiente del interno, tiene que estar } \\
\text { pendiente de los estudiantes, explíqueles, enséñeles, entonces yo sentí que mi labor como } \\
\text { docencia era más como autoritaria..." Violeta p 22, resumen de codificación NVivo }\end{array}$ \\
\hline $\begin{array}{c}\text { Facilitador } \\
\text { del } \\
\text { conocimiento }\end{array}$ & $\begin{array}{l}\text { “...en el momento de revisar los temas, uno pues tiene que saberse todo el tema completo } \\
\text { y buscar como la forma de que todos participen y que uno les pueda aportar a ellos; } \\
\text { entonces no solo como, bueno, definición, epidemiologia, clínica, sino como que uno } \\
\text { pueda escucharlos a ellos y pueda orientarlos... lo que tiene que hacer, sino guiándolo, } \\
\text { entonces bueno, mire, de este tema vamos a abordar tal y tal cosa, de pronto la literatura } \\
\text { que más nos conviene es tal, pero espero que ustedes busquen otra y que la compartamos" } \\
\text { Liliana p 6, resumen de codificación NVivo }\end{array}$ \\
\hline $\begin{array}{l}\text { Facilitador } \\
\text { de la práctica } \\
\text { clínica }\end{array}$ & $\begin{array}{l}\text { "en algún momento fue a un interno, que pregunto: ¿señora que síntomas tiene? y nuestra } \\
\text { paciente era una paciente de muchos años, del área rural, esa palabra síntoma no la tienen } \\
\text { codificada, entonces ven espera pregunta más bien, por acá ¿Qué ha sentido, que le duele, } \\
\text { que le molesta y de pronto ese orientar el abordaje del paciente también se presta no } \\
\text { solamente en el momento de compartir que conocimientos, que se ha leído, como se } \\
\text { aborda un tema, sino de pronto eso, como realizar un examen físico de pronto de una } \\
\text { manera más agradable..." Camilo p 49, resumen de codificación NVivo }\end{array}$ \\
\hline Role $n$ & $\begin{array}{l}\text { "más que todo sabes que, la seguridad, porque en ocasiones como el miedo.....como esos } \\
\text { nervios, esa angustia de no saber cómo manejar la situación, entonces eso también hace } \\
\text { parte de como la enseñanza que uno hace ahí, como que ellos al ver que uno actúa } \\
\text { tranquilo como que ya ahí dicen, bueno, realmente no es tan malo, se puede lograr se } \\
\text { puede hacer sin tener que pues llegar como al pánico o algo así, entonces también es } \\
\text { chévere.....claro ellos lo vean a uno y ven que esta uno tranquilo, igual uno les dice, o sea, } \\
\text { si llega esto tranquilos, no hay afán de tal cosa, no hay afán de tal otra y ellos poco a poco } \\
\text { se van adaptando" Camila p } 18, \text {, resumen de codificación NVivo }\end{array}$ \\
\hline $\begin{array}{l}\text { Lifelong } \\
\text { learner }\end{array}$ & $\begin{array}{l}\text { "pues hago un proceso de retroalimentación, entonces el proceso de retroalimentación va } \\
\text { a, primero que ellos me cuenten que sintieron.... lo segundo, yo siempre les pido el favor, } \\
\text { si de pronto a lo largo de la rotación que estuvieron conmigo, si les gustó la metodología } \\
\text { que tuve, si les llamó la atención algo en específico, algo que quedo volando y que no lo } \\
\text { abordamos y que pues, si o si en un momentico lo volvemos a retomar y ya por último } \\
\text { hago una retroalimentación grupal y la retroalimentación grupal va más enfocada en que } \\
\text { lo que trabajamos en equipo estuvo bien" Luisa, p 11, resumen de codificación NVivo }\end{array}$ \\
\hline
\end{tabular}

Asimismo, aquí aparecen por primera vez en las dimensiones de variación las estrategias pedagógicas centradas en el estudiante y una relación pedagógica horizontal. En contraste con las metodologías centradas en el docente, donde el profesor se considera como un "proveedor" de la verdad absoluta, las estrategias centradas en el estudiante enfatizan en la importancia del estudiante como principal gestor de su proceso de aprendizaje y al docente como un actor secundario considerado como un "facilitador" del aprendizaje, quien con su experticia y pensamiento crítico, valida la información disponible y guía a los estudiantes en la comprensión de aspectos relevantes para su práctica profesional (8).

\section{El "residente como docente" facilitador de la práctica clínica}

Al contrastar al "facilitador de la práctica clínica" con los 12 roles reportados por AMEE, este se asemeja al "docente clínico o práctico" (9). El "docente clínico o práctico" transmite información relevante para la práctica médica; para ello selecciona, organiza y 
entrega información durante diversos escenarios clínicos como la revista médica o la consulta; con énfasis en las habilidades prácticas para el ejercicio médico (9). En el estudio de Hossein sobre estilos de enseñanza, se identificó que mediante el "enseñar haciendo", el docente clínico enseña y transfiere experiencias a sus estudiantes al empoderarlos en el desarrollo de tareas variables que involucran el cuidado del paciente en un contexto clínico real; durante este acompañamiento, las tareas pueden ser realizadas por el estudiante, el docente o ambos, según la competencia del estudiante. Al hacer un paralelo con lo identificado en esta investigación y lo reportado por Hossein, el "facilitador de la práctica clínica" basa su docencia en el -enseñar haciendo- (21).

\section{El "residente como docente" role model}

Al contrastar al "role model" con los 12 roles reportados por AMEE, este es similar al "role model en el trabajo" (9). La relevancia del docente como role model ha sido documentada en diversos estudios, en donde el ejemplo brindado por el profesor en múltiples dimensiones del ser, influencia de manera significativa a los estudiantes en sus actitudes, comportamientos e incluso, en la elección de una especialidad (9). El "role model en el trabajo" ejemplifica qué se debe aprender y hacer en el contexto clínico, puesto que los estudiantes aprenden a partir de la observación e imitación de quienes admiran; acorde con la literatura, el role model es la estrategia docente más poderosa disponible para los docentes clínicos debido a que tiene un gran impacto en el estudiante al ser comparado con otras estrategias $(9,22)$.

En adición, Bandura describe al role model como aquel individuo que transmite competencias formales e informales a otros como resultado de una interacción social (ambientes familiares, sociales, profesionales o educativos) y el receptor acepta y reproduce las acciones que percibe como favorables (23-24). En términos generales, el role model influencia cada faceta del crecimiento y desarrollo del estudiante a través del ejemplo, además de ejercer funciones de motivador, guía e incluso amigo en algunas circunstancias $(8,22)$.

\section{El "residente como docente" lifelong learner}

El término lifelong learning tiene múltiples interpretaciones, pero, de forma general significa que el aprendizaje debe ocurrir en todas las etapas del ciclo vital y estar presente en todos los aspectos de la vida de una persona (academia, trabajo, hogar y comunidad); el lifelong learner tiene la capacidad de reconocer oportunidades de aprendizaje en cada persona que lo rodea, bajo cualquier circunstancia e independiente de su edad, es decir, construye habilidades y conocimiento de forma continua durante toda su vida, razón por la cual se seleccionó como nombre para esta categoría (25).

Al contrastar al "lifelong learner" con los 12 roles reportados por AMEE, no se identificó ningún rol que permita hacer una comparación directa con esta categoría (9). Sin embargo, al procurar entender al "lifelong learner" a través de lo que se ha descrito como un "buen docente" clínico, merece enmarcar algunos componentes. Si bien es difícil determinar lo que constituye un "buen docente" y no existe consenso en torno a esto, se han resaltado algunas características fundamentales identificadas en el "lifelong learner" $(9,26)$. Los "buenos docentes" clínicos conocen los principios generales de la enseñanza, tienen un buen conocimiento clínico y son capaces de correlacionar el nivel educativo del estudiante con el paciente y el contexto clínico; además, disfrutan de la enseñanza, buscan facilitar ambientes seguros de aprendizaje y son percibidos como role model altamente positivos $(9,26)$. 


\section{El "residente como docente" en potencia}

Con este estudio se destaca el valor de la identificación de las variaciones en la experiencia del rol del "residente como docente", puesto que al comprender que algunos residentes experimentan de forma más limitada su rol docente con respecto a otros (determinado por las dimensiones de variación), y que cada categoría posee sus propias ventajas y dificultades, se entiende que existe el potencial de avanzar a la siguiente categoría tras alcanzar una mayor comprensión del rol y las implicaciones de este a nivel personal, profesional y social. Entonces, se podría decir que, con los incentivos y recursos adecuados, se puede llegar a las categorías superiores de "role model" e incluso llegar a ser un "lifelong learner", categoría identificada en la cúspide de la experiencia y comparada con lo que se considera ser "un buen docente".

Por otra parte, si bien el "facilitador pasivo impuesto" afrontará mayores retos para transformar su ejercicio docente, con un adecuado entrenamiento en habilidades educativas y la promoción de contextos que potencien la motivación autónoma y el desarrollo de la identidad como educadores, es posible que se logre progresar a la siguiente categoría de descripción.

Con respecto al "facilitador del conocimiento" y el "facilitador de la práctica clínica", si bien cuentan con una identidad docente en evolución y desempeñan actividades educativas centradas en el estudiante, al no contar con entrenamiento en docencia y desconocer diversos aspectos de la importancia de su rol docente; son categorías que persisten con una marcada limitación del ejercicio de enseñanza.

Finalmente, se espera que el "residente como docente" llegue a los niveles de "role model" y "lifelong learner", puesto que integran el "ser" como finalidad pedagógica del ejercicio educativo, se reconocen a sí mismos como docentes, son capaces de sobreponerse a las barreras extrínsecas, lo cual los lleva a desempeñar un rol docente incluso en escenarios percibidos como no favorables, además de contar con motivaciones intrínsecas que trasciende su propio beneficio; condiciones muy deseables en el contexto latinoamericano donde la principal docencia es suministrada por los residentes.

Entonces, ¿Por qué no aspirar a que todos se conviertan en un "lifelong learner"? aunque a grandes rasgos estas dos últimas categorías ("role model" y "lifelong learner") son muy similares, el "lifelong learner" tiene una capacidad metacognitiva de su propio ejercicio docente, que, aunque puede ser entrenada, requiere componentes propios de la persona que sobrepasan un curso de entrenamiento de "residente como docente". Por otra parte, se encuentra el componente motivacional, propio de esta categoría y fuertemente marcada por su historia de vida, situación que valdría la pena explorar en estudios posteriores.

\section{Limitaciones}

Esta investigación corresponde a un estudio cualitativo de corte fenomenográfico, por lo que no se espera que los resultados sean generalizados a otros grupos poblacionales. De igual forma, el análisis no es absoluto y por lo tanto puede estar sujeto a múltiples interpretaciones.

\section{Conclusiones}

- Las variaciones en las experiencias del "residente como docente", están determinadas por la interacción de factores personales y contextuales, además de la propia concepción del rol docente. 
- Las categorías de descripción se encuentran interconectadas, con el potencial de avanzar hacia la siguiente categoría.

- Estos hallazgos se pueden utilizar como sustrato para el desarrollo de un programa de "residente como docente" orientado a potenciar la transformación hacia las categorías de descripción en los niveles de mayor comprensión del rol, situación deseable en el contexto latinoamericano donde los residentes tienen diversas responsabilidades docentes.

Financiación: No ha habido financiación.

Declaración de conflicto de interés: Los autores declaran no tener ningún conflicto de intereses.

Contribuciones de los autores: Todos los autores participaron en la concepción de la idea, recolección de los datos, análisis de la información y redacción colaborativa del documento final.

\section{Referencias}

1. Ramani, S., Mann, K., Taylor, D., \& Thampy, H. (2016). Residents as teachers: Near peer learning in clinical work settings: AMEE Guide No. 106. Medical teacher, 38(7), 642-655. https://doi.org/10.3109/0142159X.2016.1147540

2. Bayter, E., Cordoba, A., \& Messier, J. (2017). Residentes como docentes en la Fundación Universitaria de Ciencias de la Salud (FUCS), proyecto de residentes formadores. Repertorio de Medicina y Cirugía, 26(1), 27-34. https://doi.org/10.1016/j.reper.2017.03.001

3. Hill, A. G., Yu, T. C., Barrow, M., \& Hattie, J. (2009). A systematic review of resident-as-teacher programmes. Medical education, 43(12), 1129-1140. https://doi.org/10.1111/j.1365-2923.2009.03523.x

4. Ince-Cushman, D., Rudkin, T., \& Rosenberg, E. (2015). Supervised near-peer clinical teaching in the ambulatory clinic: an exploratory study of family medicine residents' perspectives. Perspectives on medical education, 4(1), 8-13. https:/ / doi.org/10.1007/s40037-015-0158-Z

5. Zundel, S., Stocker, M., \& Szavay, P. (2017). "Resident as teacher" in pediatric surgery: Innovation is overdue in Central Europe. Journal of pediatric surgery, 52(11), 1859-1865. https://doi.org/10.1016/i.jpedsurg.2017.05.029

6. Isenberg-Grzeda, E., Weiss, A., Blackmore, M. A., Shen, M. J., Abrams, M. S., \& Woesner, M. E. (2016). A Survey of American and Canadian Psychiatry Residents on Their Training, Teaching Practices, and Attitudes Toward Teaching. Academic Psychiatry, 40(5), 812-815. https://doi.org/10.1007/s40596-016$\underline{0491-5}$

7. Mann, K. V., Sutton, E., \& Frank, B. (2007). Twelve tips for preparing residents as teachers. Medical teacher, 29(4), 301-306. https://doi.org/10.1080/01421590701477431

8. Nawabi, S., Shaikh, S. S., Javed, M. Q., \& Riaz, A. (2020). Faculty's Perception of Their Role as a Medical Teacher at Qassim University, Saudi Arabia. Cureus, 12(7), e9095. https://doi.org/10.7759/cureus.9095

9. Harden, R. M., \& Crosby, J. (2000). AMEE guide no 20: The good teacher is more than a lecturer - The twelve roles of the teacher. Medical Teacher, 22(4), 334-347. https://doi.org/10.1080/014215900409429

10. Devine, L. A., Ginsburg, S., Stenfors, T., Cil, T. D., McDonald-Blumer, H., Walsh, C. M., \& Stroud, L. (2019). Professional Responsibilities and Personal Impacts: Residents' Experiences as Participants in Education Research. Academic medicine : journal of the Association of American Medical Colleges, 94(1), 115-121. https://doi.org/10.1097/ACM.0000000000002411

11. Hubinette, M. M., Ajjawi, R., \& Dharamsi, S. (2014). Family physician preceptors' conceptualizations of health advocacy: implications for medical education. Academic medicine : journal of the Association of American Medical Colleges, 89(11), 1502-1509. https://doi.org/10.1097/ACM.0000000000000479

12. Limberg, L. (2012). Phenomenography. In The SAGE Encyclopedia of Qualitative Research Methods (pp. 612-614). https:/ / doi.org/https:/ /dx.doi.org/10.4135/9781412963909

13. Larsson, J., \& Holmstrom, I. (2007). Phenomenographic or phenomenological analysis: does it matter? Examples from a study on anaesthesiologists' work. International Journal of Qualitative Studies on Health and Well-Being, 46 (October 2006). https://doi.org/10.1080/17482620601068105 
14. Röing, M., Holmström, I. K., \& Larsson, J. (2018). A Metasynthesis of Phenomenographic Articles on Understandings of Work Among Healthcare Professionals. Qualitative health research, 28(2), 273-291. https://doi.org/10.1177/1049732317719433

15. Stenfors-Hayes, T., Hult, H., \& Dahlgren, L. O. (2012). Three ways of understanding development as a teacher. European journal of dental education : official journal of the Association for Dental Education in Europe, 16(1), e151-e157. https://doi.org/10.1111/j.1600-0579.2011.00690.x

16. Stenfors-Hayes, T., Hult, H., \& Dahlgren, M. A. (2013). A phenomenographic approach to research in medical education. Medical education, 47(3), 261-270. https://doi.org/10.1111/medu.12101

17. Emanuel E. ¿Que hace que la investigacion clinica sea etica? siete requisitos éticos. In: Investigación en sujetos humanos: experiencia internacional Santiago de Chile: Programa Regional de Bioética OPS/OMS [Internet]. $1999 . \quad$ p. 33-46. Available from: https://www.bioeticacs.org/iceb/seleccion_temas/investigacionEnsayosClinicos/ Emanuel_Siete_Requisitos_Eticos.pdf

18. Gerlese S. Åkerlind (2005) Variation and commonality in phenomenographic research methods, Higher Education Research \& Development, 24:4, 321-334, http:/ / dx.doi.org/10.1080/07294360.2011.642845

19. Jacobs, J. C. G., van Luijk, S. J., Galindo-Garre, F., Muijtjens, A. M. M., van der Vleuten, C. P. M., Croiset, G., \& Scheele, F. (2014). Five teacher profiles in student-centred curricula based on their conceptions of learning and teaching. BMC Medical Education, 14(1), 220. https:/ / doi.org/10.1186/1472-6920-14-220

20. Jacobs, J. C. G., van Luijk, S. J., Galindo-Garre, F., Muijtjens, A. M. M., van der Vleuten, C. P. M., Croiset, G., \& Scheele, F. (2014). Five teacher profiles in student-centred curricula based on their conceptions of learning and teaching. BMC Medical Education, 14(1), 220. https://doi.org/10.1186/1472-6920-14-220

21. Hossein, K. M., Fatemeh, D., Fatemeh, O. S., Katri, V. J., \& Tahereh, B. (2010). Teaching style in clinical nursing education: a qualitative study of Iranian nursing teachers' experiences. Nurse education in practice, 10(1), 8-12. https:/ / doi.org/10.1016/j.nepr.2009.01.016

22. Cruess, S. R., Cruess, R. L., \& Steinert, Y. (2008). Role modelling--making the most of a powerful teaching strategy. BMJ (Clinical research ed.), 336(7646), 718-721. https://doi.org/10.1136/bmj.39503.757847.BE

23. Coppell, R. (2020). Revisiting the Concept of the Anti-Role-Model for Social Learning Theory in UK Education. Research Ideas and Outcomes, 6. https://doi.org/10.3897/rio.6.e60683

24. Bandura, A. (1977). Social learning theory. Englewood Cliffs, N.J: Prentice Hall.

25. Laal, M. (2011). Lifelong learning: What does it mean? Procedia - Social and Behavioral Sciences, 28(May), 470-474. https://doi.org/10.1016/j.sbspro.2011.11.090

26. Kheong Lum, S., AS Burud, I., Shirahatti, R., \& Barua, A. (2019). Students and Faculty Perspective of a Good Medical Teacher in the Asian Context. Journal of Surgery and Research, 2(3), 054-059. https://doi.org/10.26502/jsr.10020019

27. Stenfors-Hayes, T., Hult, H., \& Dahlgren, L. O. (2011). What does it mean to be a good teacher and clinical supervisor in medical education?. Advances in health sciences education : theory and practice, 16(2), 197210. https:// doi.org/10.1007/s10459-010-9255-2

28. Imafuku, R., Saiki, T., Kawakami, C., \& Suzuki, Y. (2015). How do students' perceptions of research and approaches to learning change in undergraduate research?. International journal of medical education, 6 , 47-55. https://doi.org/10.5116/ijme.5523.2b9e

29. Sjöström, B., \& Dahlgren, L. O. (2002). Applying phenomenography in nursing research. Journal of advanced nursing, 40(3), 339-345. https:// doi.org/10.1046/j.1365-2648.2002.02375.x

(C) 2021 Universidad de Murcia. Enviado para su publicación en acceso abierto bajo los términos y condiciones de la licencia Creative Commons Reconocimiento-NoComercialSin Obra Derivada $4.0 \quad$ España $\quad(C C \quad$ BY-NC-ND) (http://creativecommons.org/licenses/by/4.0/). 